\title{
A Tunable Low Noise Active Bandpass Filter Using a Noise Canceling Technique
}

\author{
N. Soltani \\ Department of Electrical Engineering, Malayer Branch \\ Islamic Azad University \\ Malayer, Iran \\ soltani.2000@yahoo.com
}

\begin{abstract}
A monolithic tunable low noise active bandpass filter is presented in this study. Biasing voltages can control the center frequency and quality factor. By keeping the gain constant, the center frequency shift is $300 \mathrm{MHz}$. The quality factor can range from 90 to 290 at the center frequency. By using a noise cancelling circuit, noise is kept lower than $2.8 \mathrm{~dB}$. The proposed filter is designed using MMIC technology with a center frequency of $2.4 \mathrm{GHz}$ and a power consumption of $180 \mathrm{~mW}$. ED02AH technology is used to simulate the circuit elements.
\end{abstract}

Keywords-Microwave Monolithic integrated circuit (MMIC; Bandpass filter; quality factor; noise cancelling

\section{INTRODUCTION}

A good Noise Figure (NF) performance in most circuit is necessary. For example, a good Low Noise Amplifier (LNA) design becomes very important because it is one of the key circuit blocks in a radio receiver system. Since the LNA can be directly added to the first stage in the receive path, it usually dominates the NF and the bandwidth in the receiver, and a variable gain LNA can improve the dynamic range of the whole receiver [1]. Especially in mobile communication or video electronic products, an LNA with good NF performance wide bandwidth and larger dynamic range in its specification is needed. Various applications of microwave frequencies have found special attention in different criteria. Tunable filters in high frequency ranges have several advantages. They can reduce the complexity of a system avoiding the introduction of filter banks. Multi band telecommunication systems, radiometers, and wideband radar systems are some of the applications of such filters. Previous tuning methods include adjusting the cavity dimensions of the resonators or altering the resonant frequency of a ferromagnetic yttrium-iron-garnet element. New approaches are based on solid state varactor diodes [2, 3]. In this paper, a tunable low noise active bandpass filter using a noise canceling circuit is designed. We propose the design of a bandpass filter in which its NF is better than 2.8 $\mathrm{dB}$ and its gain is more than $20 \mathrm{~dB}$. Two DC voltages are used to bias varactor diode in such a way that the center frequency and quality factor would be controlled using these voltages [4].

\section{FILTER STRUCTURE}

Figure 1 shows the block diagram of the desired filter. This filter is consisted of six parts: an amplifier, the LC tank, the negative conductance and the noise canceling circuit. Moreover, common gate and common drain structures are used to realize input and output impedance matching respectively. The resulted bandpass filter is shown in Figure 2. In this filter, negative conductance is added to the lossy LC tank. As a result, quality factor increases. In the proposed circuit, the feedforward noise-canceling technique $[5,6]$ is used. The circuit of the feedforward noise canceling technique is composed of an input transistor, a resistance, feedforward voltage amplifier and one adder. By using common drain and common source structure, the noise of input transistor is effectively canceled, but again in this technique the noise of the feedforward voltage amplifier and the adder is not canceled. Moreover, the noise of resistance is partly contributed in the signal.

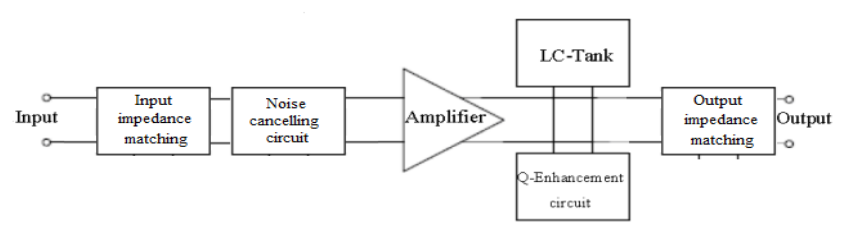

Fig. 1. Block diagram of desired filter

\section{SIMULATION RESULTS}

Simulation results are shown in Figure 3 to illustrate gain, input and output impedance matching and noise figure respectively. By inspections of these figures, one can see that the scattering parameters S11, S22 are in the desirable situation and by using the noise canceling circuit, the noise figure is kept under $2.8 \mathrm{~dB}$.

\section{FREQUENCY DISPLACEMENT}

We use the biasing voltage of varactor diodes to perform the frequency sweep. $V_{f}$ in Figure 2 biased the varactor diode and the capacity would change due to the applied voltage. This 
voltage sweeps the frequency and shifts the center frequency. The frequency displacement is about $300 \mathrm{MHz}$. The simulation results and scattering parameters are illustrated in Figure 4. By inspections of these figures, one can see that the scattering parameters are in desirable situation. The stability of this filter has been studied by Rollett coefficient (K). This coefficient is always greater than one [7]. Table I shows the magnitude of $V_{f}$ in each center frequency. Also measured quality factors and gain are presented.

\section{Noise Cancelling Circuit}

(a)

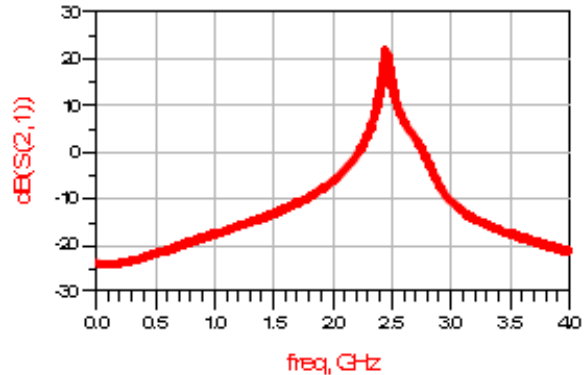

(b)

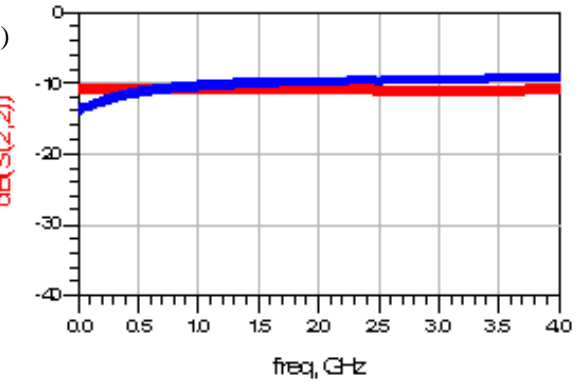

(c)

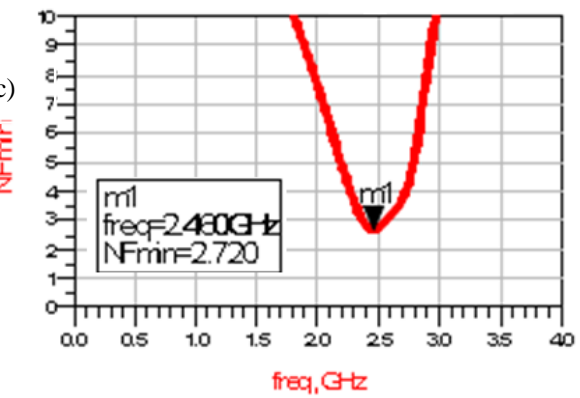

Fig. 3. Simulation results (a) gain parameter; (b) scattering parameters $\mathrm{S} 11, \mathrm{~S} 22 ;(\mathrm{c})$ noise figure
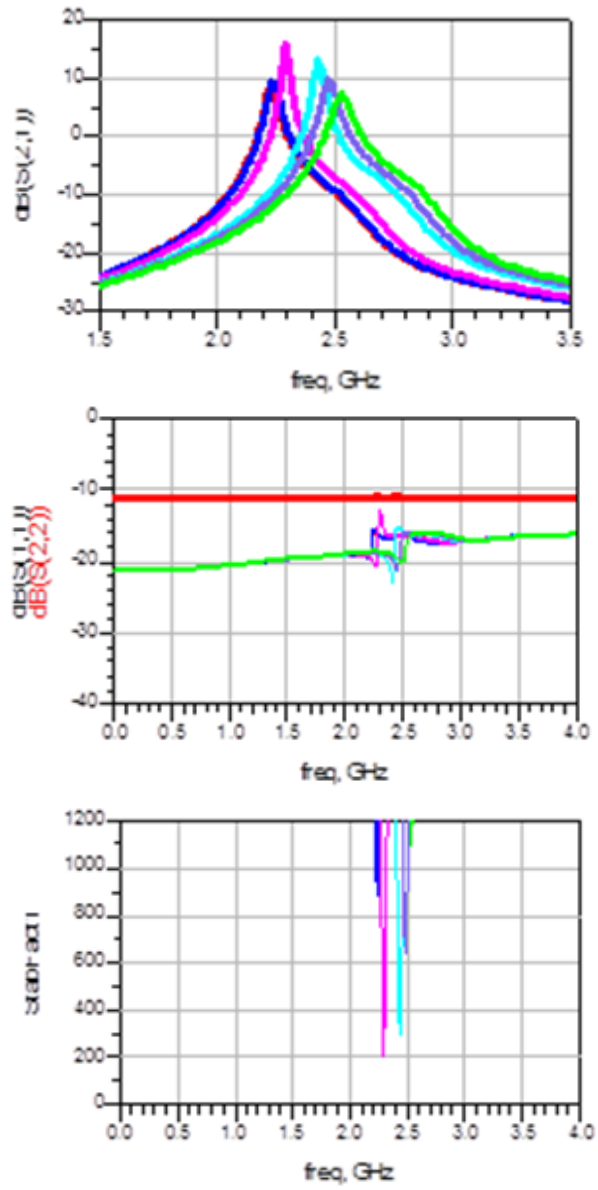

Fig. 4. Frequency displacement, scattering parameter S11 and S22 and stability factor of the low noise bandpass filter 
TABLE I. VALUE OF $V_{\mathrm{F}}$, FERECUENCY VARIATION, GAIN AND QUALITY FACTOR OF THE BANDPASS FILTER

\begin{tabular}{|c|c|c|c|}
\hline Vf1(V) & $\mathbf{f}_{\mathbf{0}}(\mathbf{G H z})$ & Gain (dB) & $\mathbf{Q}$ \\
\hline 4.2 & 2.219 & 10.02 & 43 \\
\hline 4.4 & 2.475 & 16.23 & 58 \\
\hline 5 & 2.413 & 13.05 & 53 \\
\hline 5.2 & 2.477 & 10.00 & 48 \\
\hline 5.5 & 2.515 & 8.76 & 19 \\
\hline
\end{tabular}

\section{INCREASING THE QUALITY FACTOR}

By using $\mathrm{Va}$ that is shown in Figure 2, in a constant frequency, considerable improvement of gain and quality factor would be possible. This voltage is responsible to control the quality factor. As shown in Figure 5 changing this voltage at $2.4 \mathrm{GHz}$ gives considerable increment of quality factor from 90 to 290. We ignored the frequency shift of $50 \mathrm{MHz}$ and the stability factor is also in desirable situation. Va, gain, and quality factor of each filter are shown in Table II.
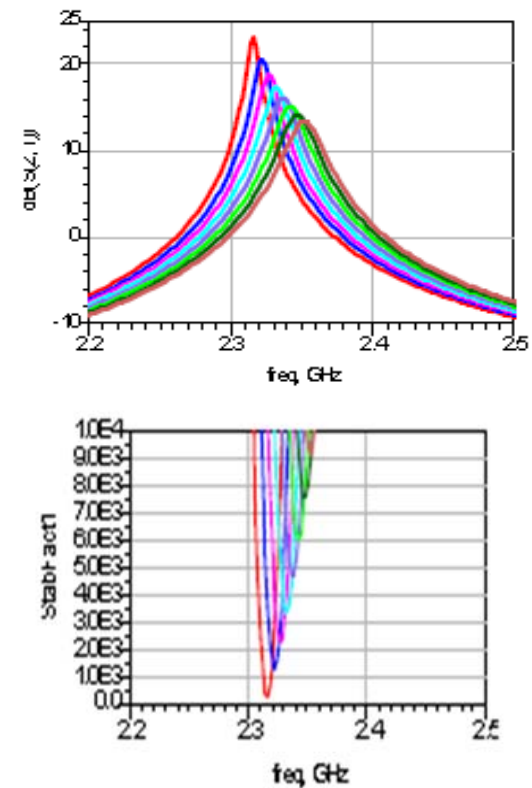

Fig. 5. Quality factor increment and stability factor at $2.4 \mathrm{GHz}$

TABLE II. VALUE OF $\mathrm{V}_{\mathrm{A}}$, GAIN AND QUALITY FACTOR OF BANDPASS FILTER

\begin{tabular}{|c|c|c|}
\hline Va1 (v) & Gain (dB) & Q \\
\hline 1 & 13.30 & 296 \\
\hline 2 & 14.02 & 219 \\
\hline 3 & 15.11 & 177 \\
\hline 4 & 15.85 & 153 \\
\hline 5 & 16.34 & 131 \\
\hline 6 & 18.00 & 118 \\
\hline 7 & 20.12 & 105 \\
\hline 8 & 22.78 & 96 \\
\hline
\end{tabular}

VI. STATISTICAL RESULTS

In this section we simultaneously exerted 5\% tolerance on the measure of 3 elements including resistor, capacitor, and transistor width in the low noise bandpass filter. These variations were completely random. Figure 6 shows the simulation results of the combination of ten different situations.
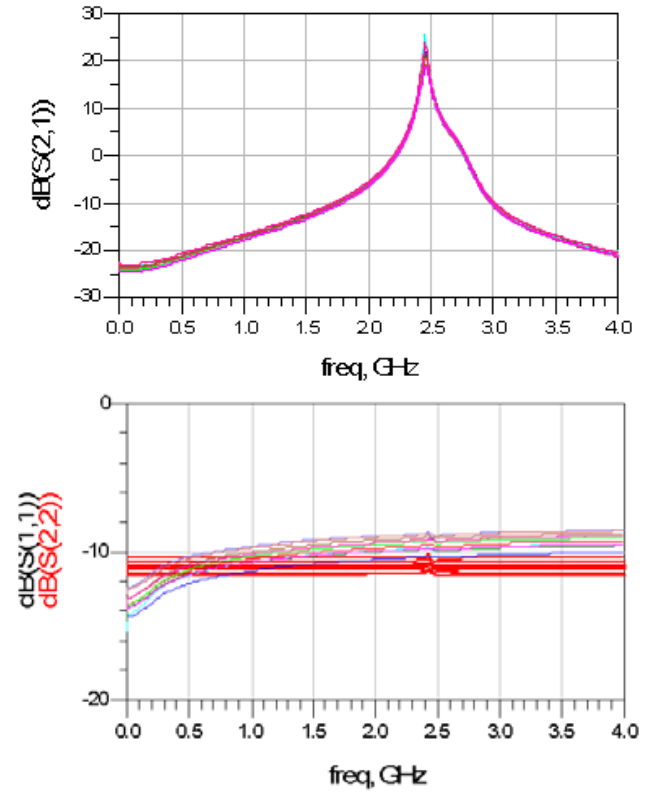

Fig. 6. Scattering parameters as a result of $5 \%$ tolerance

\section{CONCLUCION}

A tunable low noise active bandpass filter using feedforward noise canceling technique is presented. Controlling the center frequency and quality factor is investigated using two biasing voltages. The center frequency shift is $300 \mathrm{MHz}$ and Considerable improvement of quality factors is achieved (from 90 to 290). Future work should focus on the estimation of the desired biasing voltages of varactor diodes using a neural network to obtain the specific quality factor and center frequency. Also, on using active inductor to decrease the chip area. Other noise filters technologies such as BICMOS or RFCMOS should be evaluated in the same structure.

\section{REFERENCES}

[1] I. Mehr, S. Rose, S. Nesterenko, D. Paterson, R. Schreier, H. L’Bahy, S. Kidambi, M. Elliott, S. Puckett, "A dual- conversion tuner for multistandard terrestrial and cable reception”, IEEE VLSI Circuits Symp Dig pp. 340-343, 2005

[2] C .Musoll-Anguiano, I .Llamas-Garro, Z .Brito-Brito, L. Pradelll, A. Corona-Chavez, "Characterizing a Tune All Bandstop Filter", IEEE MTT-S International Microwave Workshop Series on Signal Integrity and High-Speed Interconnects, pp. 55-58, 2009.

[3] A. R. Brown, G. M. Rebeiz, "A Varactor Tuned RF Filter", IEEE Transactions on Microwave Theory and Techniques, Vol. 46, No. 7, pp. 1157-1160, 2000

[4] A. Alahyari, A. Habibzadeh, M. Dousti, "Tunable Active Dual-Band Bandpass Filter Design Using MMIC Technology”, International Journal of Engineering \& Technology, Vol. 11, No. 1, pp. 11-16, 2011

[5] F. Bruccoleri, E. A. M. Klumperink, B. Nauta, "Wide-band CMOS lownoise amplifier exploiting thermal noise canceling", IEEE Journal of Solid-State Circuits, Vol. 39, No. 2, pp. 275-282, 2004

[6] W. Keping, W. Zhigong, “A broadband noise-canceling CMOS differential LNA for $50-860 \mathrm{MHz}$ TV tuner”, International Conference on Microwave and Millimeter Wave Technology, 20-22 Dec. 2007

[7] M. Dousti, P. Langari, "A Tunable Active Recursive Filter For Communication Systems in SiGe BiCMOS”, AICT 2009. International Conference on Application of Information and Communication Technologies, 14-16 Oct. 2009 